\title{
Guidelines for the use and interpretation of diagnostic methods in adult food allergy
}

\author{
Donatella Macchia' ${ }^{1}$, Giovanni Melioli ${ }^{2}$, Valerio Pravettoni ${ }^{3}$, Eleonora Nucera ${ }^{4}$, Marta Piantanida ${ }^{3}$, \\ Marco Caminati ${ }^{5}$, Corrado Campochiaro ${ }^{6}$, Mona-Rita Yacoub ${ }^{6}$, Domenico Schiavino ${ }^{4}$, Roberto Paganelli ${ }^{7}$, \\ Mario Di Gioacchino ${ }^{8}$ and On behalf of the Food Allergy Study Group (ATI) of the Italian Society of Allergy, \\ Asthma and Clinical Immunology (SIAAIC)
}

\begin{abstract}
Food allergy has an increasing prevalence in the general population and in Italy concerns $8 \%$ of people with allergies. The spectrum of its clinical manifestations ranges from mild symptoms up to potentially fatal anaphylactic shock. A number of patients can be diagnosed easily by the use of first- and second-level procedures (history, skin tests and allergen specific $\mathrm{lgE}$ ). Patients with complex presentation, such as multiple sensitizations and pollen-food syndromes, frequently require a third-level approach including molecular diagnostics, which enables the design of a componentresolved sensitization profile for each patient. The use of such techniques involves specialists' and experts'skills on the issue to appropriately meet the diagnostic and therapeutic needs of patients. Particularly, educational programs for allergists on the use and interpretation of molecular diagnostics are needed.
\end{abstract}

Keywords: Food allergy diagnosis, Skin prick test, Molecular allergens, Molecular-based diagnosis, Challenge test, Basophil activation test

\section{Background}

Food Allergy (FA) is an increasingly recognized problem in relation to its prevalence in the general population. In Italy, it corresponds to $8 \%$ of all patients with allergies [1-3] and the broad spectrum of its clinical manifestations, ranging from mild symptoms up to potentially fatal anaphylactic shock (Table 1). FA significantly affects the quality of life of patients and their families [4]. In adults, FA may persist from childhood or may develop at an older age. In the latter case, once established, FA is maintained throughout life, while paediatric FA frequently disappears during adolescence. FA may be responsible for signs and symptoms that occur shortly after consumption of the culprit food (from a few minutes to a few hours). The earlier they arise, the more serious they are. At times, symptoms appear after physical exercise (food dependent exercise induced anaphylaxis, FDEIA) and the ingestion

\footnotetext{
*Correspondence: ma.caminati@gmail.com

${ }^{5}$ Allergy Unit, Verona University and General Hospital, Verona, Italy Full list of author information is available at the end of the article
}

(about $3 \mathrm{~h}$ before) of a specific food, which is safely eaten in the absence of exercise [5].

FA most commonly affects the skin (atopic dermatitis, urticaria, angioedema, eczema and various skin rashes) $[6,7]$. Frequently, gastrointestinal manifestations are associated with cutaneous symptoms. The gut is rarely the only organ affected by food allergy. Symptoms range from dyspepsia and meteorism to colic, diarrhoea (rarely constipation), vomiting, gastroesophageal reflux, up to the most complex malabsorption syndromes, generally due to the infiltration of inflammatory cells in the gastrointestinal mucosa [8-10]. In some cases, mainly in pollen-allergic patients sensitive to molecules homologous to those contained in specific foods, symptoms appear in the form of itching and burning of the oral mucosa, papules or vesicles in the mouth, swelling of the lips and difficulty in swallowing, being defined as oral allergy syndrome [11]. Rhinitis, conjunctivitis, asthma and laryngeal edema are all possible FA manifestations independent from sensitization to inhalant allergens [12].

Each year 4-5/100,000 patients experience an anaphylactic shock, with a cumulative risk equal to $0.5-2 \%$ [13]. 
Table 1 Main food allergy symptoms

\begin{tabular}{ll}
\hline Organs and systems & Clinical manifestations \\
\hline Respiratory & Oculorhinitis \\
& Bronchial asthma \\
Skin and subcutaneous tissue & Oedema of the glottis \\
& Erythematous rash \\
& Itching without rash \\
& Urticaria-angioedema \\
& Atopic dermatitis \\
Gastro-enteric & Eczema \\
& Oral Allergy Syndrome \\
& Abdominal pains \\
& Vomiting \\
Cardiovascular system & Diarrhoea \\
& Hypotension \\
& Cardiac arrest \\
& Anaphylactic shock \\
\hline
\end{tabular}

Foods are the main cause of anaphylactic shock for children and young adults, whereas for older people, insect stings are mainly responsible. This syndrome is due to the involvement of the cardiovascular system with a drop in blood pressure due to vasodilation and leakage of fluids from the circulation, with systemic consequences [14]. The term anaphylaxis (without shock) is referred to a reaction involving multiple organs, usually the skin, gastrointestinal tract and respiratory system.

There is no consensus on allergy due to food contaminant and additives. Clinicians sometimes report the disappearance of the characteristic symptoms of food allergy after an additive-free diet, despite the fact that there is no scientific evidence on their actual role in causing symptoms [15-17]. In any case, reactions are not mediated by an immunological mechanism and are classified as nonallergic hypersensitivity reactions. There is a possibility that food reactions also stem from some non-protein food component or from other mechanisms, for example, cell-mediated mechanisms. These include reactions to orally ingested nickel, the so-called Systemic Nickel Allergy Syndrome, which is characterized by the appearance of gastrointestinal symptoms (typically meteorism, colic and diarrhoea) and skin manifestations (eczema, urticaria and angioedema) in sites without nickel contact in patients with nickel contact dermatitis, and responds positively to a low-nickel diet [18].

Diagnostic efforts are directed to the identification of the food(s) involved in triggering and/or maintaining the symptoms. This can be achieved by using all available diagnostic methods applied in an appropriate sequence, avoiding non-standardized ones.
The purpose of this document is to define guidelines for the use and interpretation of scientifically validated and recognized diagnostic methods for food allergy.

\section{Basic concepts of FA}

Primary forms of FA are due to a sensitization process caused by ingestion. In the secondary forms, the patient is sensitized by inhalation to allergens containing molecules homologous to those contained in certain foods, whose ingestion may cause symptoms usually in the oral cavity, in the frame of an oral allergy syndrome [19]. Several molecules with different characteristics act as food allergens. Some of them are stable, enduring heating, cooking, storage and digestion (linear epitopes), while others are less stable (conformational epitopes) losing their allergenicity in cooking and preservation [20]. The patient with FA can be sensitized to both labile and stable components. The stability/lability to physical agents (heat, gastric $\mathrm{pH}$, enzymes like protease, pepsin and so on) is a requisite for an allergen to interact with the IgE antibody. Thus, a component sensitive to heat will be virtually absent in a cooked food, while a determinant resistant to heat, $\mathrm{pH}$ and peptidase (for example, Lipid Transfer proteins-LTP) will reach the bowel practically intact despite cooking and passage through the gastric and pancreatic digestion [21]. A particular situation arises with the use of antacid drugs that do not allow (or partially allow) the denaturation of acid sensitive proteins, thus resulting in unpredictable symptoms [22]. Other substances can act as "co-factors", increasing the likelihood of anaphylaxis from food allergens. They include alcohol, non-steroidal anti-inflammatory drugs (NSAIDs), hormonal influences, bacterial or viral or parasitic infections [23] and chemicals [24-26].

The large variety of clinical manifestations (Table 1) and the complexity of allergens often make the diagnosis of FA difficult. A component-specific profile, other than extract-specific, should be used for an optimal definition of the sensitization. In addition, in the specific field of food allergy, it is crucial to discriminate between crossreactions and co-sensitization, particularly for members of the plant kingdom ("pollen food allergy"), and to more accurately estimate the risk of severe reactions. The sensitization to cross-reactive molecules is relatively rare in childhood but tends to appear during adolescence and remains stable in adults. The recent adoption of individual allergenic molecules (Molecular-Based Diagnosis, MBD) in diagnosis allows for the definition of a more precise IgE profile for the patient, e.g. adding prognostic information related to the possible risk profile of the reaction. Understanding the fine relationships between the results of in vivo and in vitro tests and the 
patient's clinical picture is the key for any further clinical decisions.

\section{Diagnostic methods for food allergy}

A correct diagnosis of FA requires an established diagnostic procedure. The first step is always the patient's history aimed at identifying the suspected relevant food(s) and the relationship between the ingestion of a specific food and the occurrence of symptoms. Then, the dependence of clinical manifestations from an immune mediated reaction must be assessed. This can be done by both in vivo and in vitro tests.

The standardized diagnostic methods are classified into first, second and third level.

\section{First-level methods}

\section{Medical history}

Medical history is essential in every field of medicine because it allows one to obtain all the information and data that can help to move towards the diagnosis of a certain disease. It comprises the physiological, family (investigating all possible genetic risk factors or any family predisposition) and the past and current medical history. The latter investigates the disorder for which the patient consulted a doctor. In suspected FA, repeated clinical manifestations related to the ingestion of given meals are highly indicative. Medical history should be addressed to clarify (a) the presence or absence of similar symptoms in other people when they consumed the same food(s); (b) the ingested food(s) in the 2-4 $\mathrm{h}$ before the onset of symptoms; (c) the allergens that may contaminate food preparation (for example, casein, latex, ovomucoid); (d) the cooking and storage of food; (e) the presence of triggering factors; (f) the existence of other allergies (such as respiratory or skin allergy) or other diseases [27]. A correct diagnostic approach also requires a complete physical examination. When too much time has elapsed from the appearance of symptoms, it could be difficult to identify the offending food, in particular, if the allergen is not easily identifiable or "hidden". Medical history can be reevaluated starting from the results of in vitro and in vivo tests, which could demonstrate sensitizations to foods that were initially not considered $[28,29]$.

\section{Skin tests}

Skin prick test (SPT) The SPT is a well-standardized, simple, cheap and low-risk diagnostic test. It should be the first step performed and both inhalant and food allergens should be tested. Table 2 shows a panel of food allergens to be tested, supplemented, where appropriate, by foods chosen according to patient's history and dietary diaries, and Table 3 shows the technical procedure to be used. The SPTs to foods have a low specificity with a low
Table 2 Food panel for Prick test

\begin{tabular}{ll}
\hline Egg & Peach \\
Peanut & Apple \\
Beta-lactoglobulin & Cod \\
Banana & Hazelnut \\
Carrot & Walnut \\
Casein & Fish \\
Bean & Pea \\
Wheat flour & Chicken \\
Shrimp & Tomato \\
Lactalbumin & Rice \\
Pork & Celery \\
Corn & Soybeans \\
Almond & Egg yolk \\
\hline
\end{tabular}

positive predictive value. Thus, a positive result, unless confirmed by the clinical data, does not allow for a definitive diagnosis of FA [28-30]. In children, cut-off values for the SPT reaction diameter for certain food allergen (milk: $8 \mathrm{~mm}$, egg: $7 \mathrm{~mm}$, peanut: $8 \mathrm{~mm}$ ) have been identified but are not universally acknowledged. However, oral food challenges were always positive (100\% specificity) in children with cutaneous reactions of this diameter or above [31, 32]. In general, SPT have an excellent sensitivity with high negative predictive value $(>90 \%)$, thus a negative result generally rules out the possibility of an IgE-mediated sensitization. However, this is true only for foods containing stable proteins, such as casein from cow's milk, egg ovomucoid, albumin and peanut vicilins, which are well represented in the extract. The SPT performed with allergenic extracts containing thermolabile molecules, such as pathogenesis-related-10 (PR-10) proteins have a low negative predictive value. For these allergens, the prick + prick $(\mathrm{P}+\mathrm{P})$ procedures with fresh foods can be useful.

The major limitations of allergen extracts for SPT are represented by (a) the content, because each extract is a heterogeneous mixture composed of major and minor allergenic proteins, and other biologically inactive components such as non-allergenic proteins, glycoproteins and carbohydrates, (b) the production process, because some allergens may undergo partial degradation during the extraction, (c) the cross-reactions, as different biological sources may contain cross-reactive allergens.

An in vivo MBD approach (available in vitro for many molecules, shown in Table 4) is also possible with extracts containing high concentrations of LTP (a gastro- and thermo-stable protein from Rosaceae) and palm profilins (Pho d 2, an ubiquitous gastro- and heat labile plant protein). Their use, to complete the diagnostics performed with extracts from whole sources, allow for a 
Table 3 Technical procedure for SPT

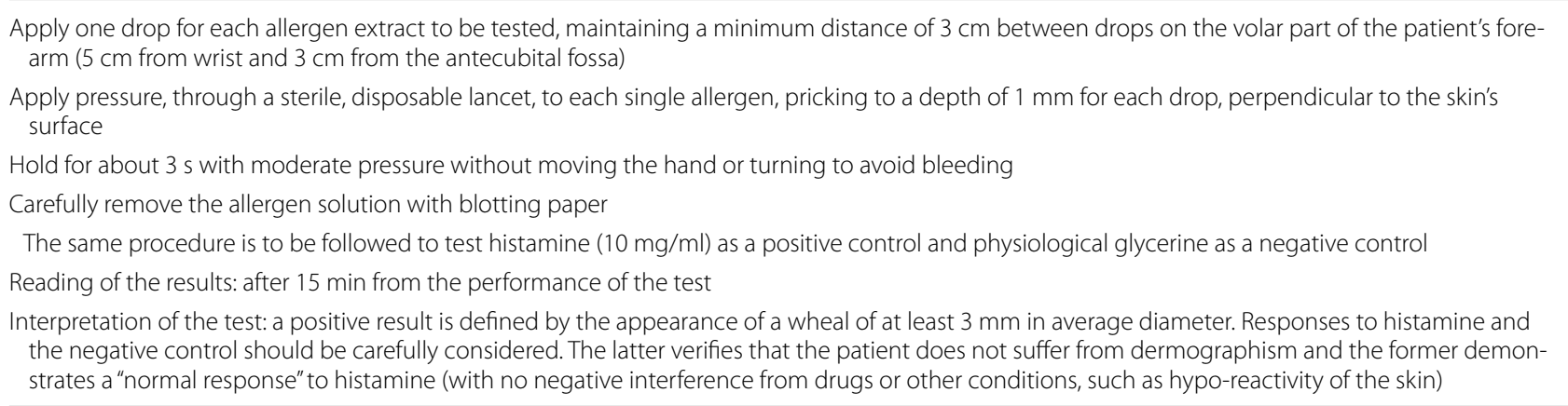

Table 4 Native or recombinant molecules available for SPT

\begin{tabular}{ll}
\hline Molecule & Source \\
\hline Lactalbumin & Cow's milk \\
Beta-lactoglobulin & Cow's milk \\
Casein & Cow's milk \\
Ovalbumin & Egg white \\
Ovomucoid & Egg white \\
LTP (Pru p 3) & Peach \\
Profilin (Pho d 2) & Palm tree \\
PR10 (Mal d 1) & Apple (not available in Italy) \\
\hline
\end{tabular}

more precise assessment of the ingestion risk of the suspected food [33].

Prick + prick $(P+P) \mathrm{P}+\mathrm{P}$ is performed with fresh food, in particular vegetables, when the commercial extract is negative (or unavailable) but the clinical history is suggestive. When the food is solid, the technique involves firstly puncturing the fresh food (some allergens are located just under the skin of the fruit) and then the patient's skin with a lancet according to the SPT standard procedure [34]. When the food is liquid, the technique is the same as in SPT.

$\mathrm{P}+\mathrm{P}$ has a good diagnostic reliability [35] with high predictive negative values. In the case of a positive result, it must be always taken into account that some foods are rich in histamine and lectins and can produce false positives. Obviously, the use of skin P $+\mathrm{P}$ with fresh food is not entirely risk-free and highly sensitive subjects may suffer systemic adverse reactions [36].

\section{Atopy patch test (APT)}

The APT is performed through the same technique used for common patch testing to identify the responsible hapten in contact dermatitis, and is aimed at assessing the delayed cell-mediated hypersensitivity to foods that may especially occur in children with atopic dermatitis or gastrointestinal reactions to foods. In 2010, the APT was considered an emerging test, like BAT and MBD [37], but subsequent studies did not confirm its diagnostic role to be as important as the other two techniques.

\section{Second-level methods \\ In vitro assays for total serum IgE (tlgE) and specific IgE (slgE) to foods}

Like SPT and P + P, in vitro tests only certify a sensitization and the interpretation of results is the allergist's task. Thus, measuring tIgE may be useful in grading allergy conditions, but only when used in combination with other tests. Indeed, $\operatorname{tgE}$ alone has no predictive value in relation to the diagnosis of FA. The assay of sIgE for food extracts is a second-level test in the view of costs. Thus, it should be requested only after skin tests. However, it may still be exceptionally considered a first-level test in those conditions in which SPT cannot be performed (e.g. very young paediatric patients, concomitant antihistamine therapy or skin alterations, risk of systemic reactions). Importantly, an in vivo test is able to detect the biological effects (revealed by wheal, redness, itching, etc.) caused by the presence of sIgE bound to skin mast cells, while the serum test only detects the presence of circulating IgE specific to a particular allergen. It is therefore possible that the results of the two tests are different [38].

Nowadays, quantitative methods with extracts have levels of sensitivity (and negative predictive values) comparable to APT, with high specificity and positive predictive value [37]. The test is suitable to detect the IgE specific for a given allergen, in a quantitative way, in a range between 0.10 and $100 \mathrm{kU} / \mathrm{L}$. As for the SPT reaction diameter, specific IgE levels exceeding a certain value ("diagnostic cut-off") showed a predictive value of $95 \%$ for a symptomatic allergy [32, 39] (Table 5). Thus, in the presence of a compatible clinical history, sIgE can confirm the diagnosis of FA without requiring further challenge tests. However, the predictive values vary from one study to another. The results are influenced by many variables such as the patient's age, duration of food allergen avoidance at the time of testing, selection of patients 
Table 5 Sensitivity, specificity, positive (PPV) and negative (NPV) predictive value of tests for the detection of specific lgE in vitro for the most common food allergens

\begin{tabular}{|c|c|c|c|c|c|}
\hline Allergen & Sensitivity (\%) & Specificity (\%) & PPV (\%) & VPN (\%) & Diagnostic cut-off (kUA/l) \\
\hline Egg & 61 & 95 & 98 & 38 & 6 \\
\hline Milk & 57 & 94 & 95 & 53 & 15 \\
\hline Peanut & 57 & 100 & 100 & 36 & 14 \\
\hline Codfish & 63 & 91 & 56 & 93 & 3 \\
\hline Soybean & 44 & 94 & 73 & 82 & 30 \\
\hline Wheat & 61 & 92 & 74 & 87 & 26 \\
\hline
\end{tabular}

and clinical disorders, and have been validated on nonEuropean test subjects. It is also important to stress that the values of specific $\operatorname{IgE}<0.10 \mathrm{kU} / \mathrm{L}$ does not exclude the possibility of an IgE-mediated allergic reaction and that the confirmation of a negative test, in the case of strong clinical suspicion, can only be achieved with negative SPTs and negative challenge tests.

\section{In vitro $M B D$}

Diagnostics based on allergenic extracts allow for the identification of the allergen source (e.g. fish, egg, milk, etc.) but not the molecular component to which a patient is sensitized, which can be studied instead through in vitro $\mathrm{MBD}$ and therefore used to improve the result of a sIgE test [20]. In vitro MBD uses molecular allergens isolated from a given allergen source (purified or native allergens) or produced by recombinant DNA technology (recombinant allergens) (Tables 6, 7). This approach improves the description of the $\operatorname{IgE}$ repertoire against food allergens or their molecular components and explains cross-reactions and their role in FA.

The MBD approach should be used to distinguish patients with genuine sensitization towards a food (with high risk of accidental ingestion) from those with co-sensitization, i.e. sensitization to ubiquitous proteins present in pollen (which act as primary sensitizing) and also common in food (with a much lower risk of adverse reaction). Again, it is possible to identify patients characterized by sensitization to food independently by a sensitization to aeroallergens (primary sensitization) and patients with a "pollen-food syndrome", where the first sensitization occurs via inhalation and the great homology between the allergen of the "first sensitizer" and some food allergens is responsible for the patient's symptoms presenting as an oral allergy syndrome [40, 41].

Identifying cross-reactions is a further benefit of MBD. The allergist is able to understand whether a single, a few closely related or several widely different food allergen sources should be considered in a dietary approach. The allergist will also be able to assess the risk of a given FA identifying, by in vitro MBD, patients sensitized to "relatively harmless" or potentially very dangerous components [20] that need the prescription of life-saving drugs such as auto-injectable adrenaline together with a strict allergen avoidance. The use of MBD requires allergists to acquire new skills.

Primarily, they need to learn the new allergen nomenclature [42, 43]. International classification ranks the allergenic source first by its scientific name, from which it takes the first three letters of the generic name and the first letter of the species (or two letters when confusion is possible): e.g. apple is scientifically called "Malus domestica": therefore Mal d indicates the allergen source. Adding a number (1, 2, 3 etc.) indicating the chronological order of the identification allows for the classification any allergenic molecules: e.g. for apple the identified molecules are named Mal d 1, Mal d2, Mal d 3, and Mal d 4.

It is also important to know the molecular allergenic content of foods. Some molecules are specific for a given food, allowing the identification of the primary sensitizer, others share common epitopes (antibody binding sites) and the same IgE can induce an immune response to allergenic molecules with similar structures from different allergen sources [33]. In the example of apple, Mal d 3 is an LTP molecule homologous to the LTP of peach, nuts, apricot, cherry, etc. and an exclusion diet should prohibit all these foods, but only according to the patient's history [44]. Indeed, Mal d 1 is highly homologous to the birch pollen allergen Bet $\mathrm{v} 1$ and characteristically induces an oral allergic reaction [45].

The molecular structure and physiochemical properties of allergens are major determinants of their clinical relevance. For example, LTPs are particularly resistant to high temperature and enzymatic degradation, so cooking and digestive processes are unable to deactivate their allergenic capacity. For this reason, LTP exposure through the gastrointestinal tract may induce sensitization in predisposed individuals and may trigger severe reactions in sensitized patients [46]. The specific patient's sensitization profile is relevant in terms of risk assessment. In 
Table 6 Major food allergens and components available for molecular diagnostics using ImmunoCAP (or ImmunoCAP ISAC)

\begin{tabular}{|c|c|}
\hline Allergens (or allergen source) & Protein family \\
\hline \multicolumn{2}{|l|}{ Cupin superfamily } \\
\hline Vicilins & Ara h 1 (peanut) \\
\hline Legumins & Ara h 3 (peanut), Cor 9 (hazelnut) \\
\hline \multicolumn{2}{|l|}{ Prolamin superfamily } \\
\hline 2S albumin & Ber e 1 (brazil nut), Ara h 2 (peanut), Gly m 6 \\
\hline Lipid transfer protein (LTP) & Pru p 3 (peach), Cor 8 (hazelnut), Art v 3 (Composite) Jug r 3 (walnut) \\
\hline Cereal prolamines & Tri 19 (wheat) Tri a 14 \\
\hline \multicolumn{2}{|l|}{ Pathogenesis-related (PR) proteins } \\
\hline PR10: intracellular proteins & Pru p 1 (peach), Api g 1 (celery), Gly m 4 (soy) \\
\hline PR3: chitinase Class 1 & Hev b 11, Hev b 2.6 (latex, banana, avocado) \\
\hline Profilins & Pru p 4 (peach) (Bet v 2, Phl p 12, Hev b 8) \\
\hline Cross-reactive carbohydrate determinants & MUXF3 (celery, tomato) \\
\hline Tropomyosins & Pen a 1 (shrimp) \\
\hline \multicolumn{2}{|l|}{ Calcium binding proteins } \\
\hline Parvalbumin & Gad c 1 (codfish) \\
\hline Milk proteins & Bos d 4 ( $\alpha$-albumin), Bos d 5 ( $\beta$-lactoglobulin), Bos d 8 (casein), Bos d lactoferrin (lactoferrin) \\
\hline \multirow[t]{4}{*}{ Egg protein } & Gal d 1 (ovomucoide) \\
\hline & Gal d 2 (ovalbumin) \\
\hline & Gal d 3 (conalbumin) \\
\hline & Gal d 4 (lysozyme) \\
\hline
\end{tabular}

\section{Table 7 Families of protein carbohydrate molecules mainly involved in food allergy}

Molecules associated with allergy to food source (or source allergen)
PR-10 proteins (homologous to Bet $v$ 1)
Non-specific lipid transfer proteins (nsLTP)
Profilins
Storage proteins
Thaumatin-like-proteins (TLP)
Cross-reactive carbohydrate determinants (CCD)
Molecules associated with allergy to food of animal origin
Tropomyosins
Parvalbumins
Caseins
Lipocalin, Family of lysozyme, Family Transferrins, Ovomucoids

fact, the simultaneous sensitization to peach LTP Pru $\mathrm{p}$ 3, Pru p 1 and Pru p 4 in the same patient seems to exert a protective role in comparison with Pru p 3 sensitization alone, as it is associated with less severe symptoms [47]. Similarly, it has been recently shown that in peach-allergic patients with tomato hypersensitivity, sensitization to rPru $\mathrm{p} 3$ seems to be a surrogate biochemical marker for a severe tomato allergy, whereas the presence of anti-rPru p 1 IgE may be an indicator of a mild tomato allergy [48].

Profilins are pan-allergens (present in many plant species not botanically related) protease sensitive and less heat sensitive that mainly induce an oral allergy syndrome, while severe reactions are rare [49].

Therefore, the allergist approaching the MBD should know the chemical, physical and immunological characteristics of all allergenic families, their biodegradability, cooking/heat resistance/sensibility etc. The stability/ lability of a molecule (along with the clinical history) helps the clinician to evaluate the risk of systemic versus local reactions. Stable allergens are generally associated with severe systemic reactions, whereas labile allergens are associated with low/mild reactions and cooked food is often tolerated.

Moreover, it is essential to know to which family the various molecules belong and their structural similarity within the family (generally characterized by a greater than $50-70 \%$ sequence homology).

In the above-mentioned example of apple, MBD can distinguish between fruit allergy due to LTP sensitization and a pollen-related apple allergy. Sensitization to Mal d 3 (an LTP protein) indicates a fruit allergy where peach is often the primary sensitizer $[50,51]$. Sensitization to Mal d 1 (a PR-10 protein) is seen in birch-pollen allergic patients and is caused by crossreactivity with the main birch allergen Bet v $1[52,53]$. The presence of IgE antibodies to profilin (e.g. Mal d 4 , homologous of $\mathrm{Phl} \mathrm{p} \mathrm{12)} \mathrm{is} \mathrm{indicative} \mathrm{of} \mathrm{an} \mathrm{apple}$ allergy related to a grass-pollen sensitization [53, 54]. 
Patients with IgE antibodies to Mal d 2 and 3 (LTP stable proteins) are at higher risk of developing systemic reactions. IgE antibodies to Mal d 1 and/or profilin and not to Mal d 2 and 3 suggest that predominantly local oral symptoms may occur. Apple-allergic patients sensitized to Mal d 3 may tolerate peeled apples. Appleallergic patients sensitized to Mal d 1 and/or profilin (that are labile proteins) may often tolerate cooked apples.

MBD is a complex area, but as it provides new and relevant information for the allergist, it will soon become a standard tool for the diagnosis of FA. Educational programs for allergists on the use and interpretation of MBD are needed [55].

In vitro $M B D$ is defined as single or multi-plexed IgE assay microarray. By the single-plexed diagnostics the choice of the components to be tested is relies on the allergist's judgment, based on the patient's SIgE profile. In poly-sensitized patients, a complete recognition of the IgE profile might require a large number of assays. In these cases, it may be reasonable to use the multi-plexed allergen microarray (AMA) that allows for the detection of specific reactivity to over 100 allergen components. The most popular form (the ImmunoSorbent Allergen Chip-ISAC) currently contains inhalants, foods, latex and insect venom. Despite AMA not being a quantitative assay, the correlation between the results of microarrays and the results of sIgE tests are largely super-imposable. Thus AMA is suitable in both paediatric and adult serum samples when the number of molecular components to be tested using single-plexed methods is too high to be cost-convenient or when the need for extensive research of sensitization is required [20]. This is particularly true in highly complex patients presenting symptoms of a cross-sensitization to inhalant and food and clinical evidence of food allergy. AMA is a powerful in vitro test that requires specific expertise but provides a very large amount of information to the allergist.

MBD diagnostics, especially microarray, are expensive compared with traditional tests, unless a single test is considered. Economic considerations may influence the decision of using a single or multiplex approach in individual patients. Using the microarray diagnostics allows for the performance of a broad-spectrum analysis of a patient's IgE profile with a small blood sample. It may reveal unanticipated sensitivities, possibly to potentially harmful molecules, making the interpretation of such sensitization difficult in the case of a clinically silent history, but giving the allergist the chance to investigate other hypersensitivities and to alert the patient towards possible risks. This clearly demonstrates that in vitro diagnostics, including $\mathrm{MB}$, should be evaluated within the framework of a patient's clinical history, since allergen sensitization does not necessarily imply clinical responsiveness.

\section{Third-level methods \\ Oral provocation test (OPT)}

OPTs are the most reliable tests in the diagnosis of clinically relevant IgE associated food allergies once allergen specific IgE has been detected. The OPT remains the "gold standard" to establish or exclude the liability of a particular food in causing an adverse reaction [56-61]. The actual value of this method is its functional result. Indeed, only foods causing a clinical evidence of allergy are considered positive. When first and second-level methods have been unable to indicate the food that is responsible for the symptoms, the clinical relevance of a detected sensitization may also be investigated by a targeted elimination diet to perform before the OPT [28]. Furthermore, if multiple triggers are suspected, the elimination diet can help in selecting food to be tested through the OPT, which remains the most important diagnostic tool in food allergy diagnosis. Once the diagnostic workup has been concluded, the elimination diet of the culprit food/s usually represents the treatment for known food allergies, as well as educating the patient about proper food preparations and the risks of occult exposure [28, 29]. Ongoing investigations are currently evaluating the role of food immunotherapy as a potential FA therapy, to be performed by highly skilled specialists in appropriate settings [29, 62].

The OPT is a third-level procedure that should be carried out when previous diagnostic levels were unable to give sufficient information for the clinical diagnosis [60]. During the patient follow-up, OPT is useful in detecting an acquired tolerance for the specific food. The functional identification of the causative food allows one to avoid its assumption as well as the establishment of unnecessary rigid diets. Due to the potential risk of severe adverse events, the test has to be performed in a hospital setting with personnel trained in resuscitation procedures and the availability of emergency drugs.

The indications of OPT $[61,63-65]$ are: (a) to identify the food responsible for acute reactions, or to monitor the unexpected tolerance in case of a history of allergy; (b) to determine the offending food in chronic conditions such as atopic dermatitis or eosinophilic esophagitis; (c) to expand the diet in subjects with multiple dietary restrictions; (d) to establish the degree of tolerance to cross-reactive foods and to establish possible acquisition of a spontaneous tolerance to food.

The contraindications are: (a) previous severe anaphylactic reactions (especially recent); (b) level of specific IgE exceeding the cut-off for which there is a high probability 
that the oral test is positive; (c) reactivity to individual molecules identified with the MBD that indicate a possible severe reaction; (d) reactions occurred during the performance of the SPT and (e) a progressive systemic disease, in particular when the patient is taking medications that could mitigate (antihistamines, corticosteroids) or amplify ( $\beta$-blockers, ACE inhibitors, NSAIDs, etc.) the reaction $[63,64]$.

The test consists of gradually increasing doses of the appropriately diluted food, starting from the lowest dose and checking the presence of relevant symptoms. The test can be performed in three different settings [63]:

Open OPT is used for immediate reactions when the risk of severe reaction is reduced. It can be performed on an outpatient basis with a simplified protocol of administration and an observation time of about $2 \mathrm{~h}$. It can be strongly influenced by the age and by the subject's psychological behaviour. If negative, the food can be reintroduced into the diet. In the case of suspected positive reaction, it should be checked in a double-blind OPT setting.

Single-blind placebo OPT (SBP-OPT) it consists of two sessions, one with placebo and one with the suspected food. When a strong psychological component is suspected, the placebo should be tested first. The patient undergoing the SPB-OPT is informed that the food may or may not be present in the administered dose. If the answer is negative or positive symptoms are observed, it is not necessary to continue the investigation. Repeated sessions with placebo or suspected food are useful for the confirmation of vague symptoms. In the case of positivity with placebo, a DBP-OPT will be necessary. In the case of a negative result, the tolerated food must be ingested in its natural form $2 \mathrm{~h}$ after or on the day after the test. The tolerance should be checked with follow-up.

Double-blind placebo controlled test (DBPCT) the gold standard. The foods to be tested are prepared by professional personnel not involved in the clinical examination. Placebo and food must have a very similar look and taste. Only when the test is completed can the doctor and patient know the pattern of administration and discuss the results [66].

\section{Fourth-level methods}

\section{Basophil activation test (BAT)}

The BAT can be used in the study of IgE (and non-IgE) mediated allergic reactions $[67,68]$. The rationale of this test is the change in the phenotype of activated basophils after in vitro incubation of the patient's whole blood with the allergen. The BAT is a useful complementary tool to the in vitro diagnosis of FA caused by milk, egg, peanut and wheat $[69,70]$ when IgE may be involved.
Interestingly, a recent study of 20 peanut-allergic children showed that when basophils were stimulated with decreasing doses of allergens until threshold sensitivity was reached, 19 were negative to peanut but 17 were positive to $\mathrm{rAra} h 8$, suggesting that the children sensitized to Ara $\mathrm{h} 8$ but not peanut storage proteins may be at risk of systemic allergic reactions, especially when eating large amounts of peanuts [70].

Reactions unrelated to IgE may also be assessed by BAT, as evidenced for wine and beef [71, 72]. Recently it was also used in the decision-making process for the reintroduction of milk in children allergic to casein [73]. Today, BAT is the only assay that mimics, in the test tube, what happens in vivo. After an extensive validation, BAT should distinguish sensitization from a clinical allergy. The method still suffers some critical issues that can make it a routine test only in specialized laboratories (Fig. 1).

\section{Complementary alternative tests}

Frequently, patients undergo complementary/alternative tests after a negative response to a common diagnostic work-up or when non specific symptoms predominate (e.g. migraine, abdominal discomfort, chronic urticaria or other skin abnormalities, chronic fatigue, weight gain or lack of success in weight loss diets), which are erroneously classified as "food allergy" [74]. It represents a common diagnostic label suggested by physicians without specific expertise in the field of FA mechanisms and food-related disorders $[75,76]$.

The most common (not validated) alternative diagnostic techniques are:

In vivo:

- Electrodermal tests: they measure the change in the skin's electrical conductance once the subject has been exposed to an allergenic substance through specific devices [75].

- Kinesiology: it registers the decreased strength of muscular contraction related to contact with an allergen [75].

- Provocation/neutralization testing: it identifies the onset of "untoward effects" provoked by the administration (intradermal or sublingual) of allergenic substances [76]. The same technique is used as a therapeutic tool.

In vitro:

- Leukocytotoxic tests: they detect the shape/volume abnormalities of peripheral leukocytes when an allergen in a solid and not measurable phase comes into contact with them [77]. 


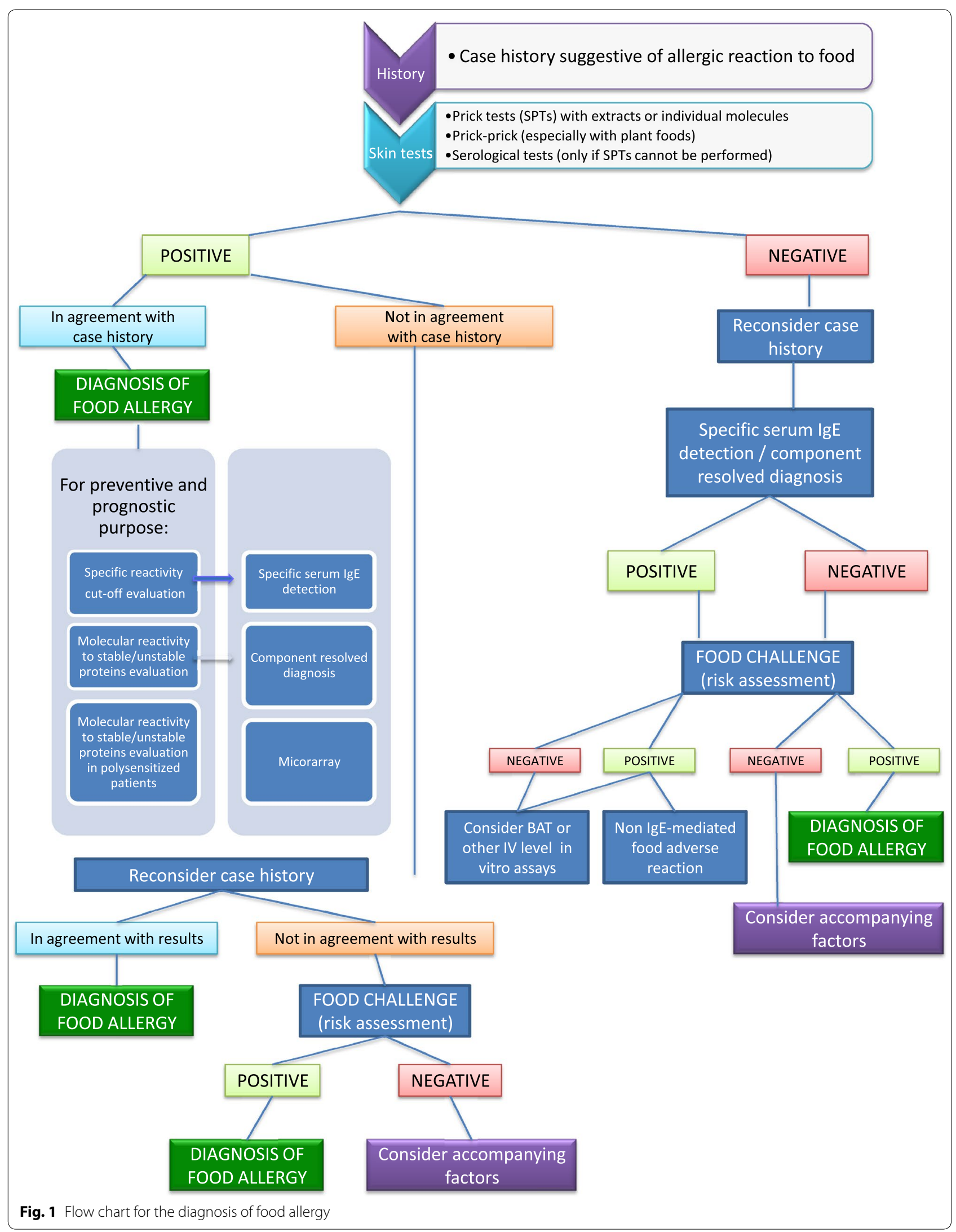


A direct comparison between such tests and gold standard methods has so far failed in all cases to demonstrate their validity [78]. Their use is strongly discouraged.

\section{Conclusion}

The diagnosis of FA is an integrated procedure that can be carried out in different steps (Fig. 1). Some patients can be diagnosed easily by the use of first- and secondlevel tests, while complex patients, with poly-sensitization and pollen-food syndromes, frequently require a third-level approach. In recent years, the diagnostic assays for FA have been significantly expanded and standardized tools and procedures are now available to the allergist.

Currently, demanding issues are related to FA diagnosis: (1) Identified food(s) should be excluded from the diet; (2) the patient must be properly informed about the relative risk of ingesting the sensitizing foods, even inadvertently as hidden foods in different preparations; (3) the allergist should explain all preventive and curative measures to be taken in case of allergic reactions, including potential medical urgency. In particular, the patient must be informed of the possibility that certain concurrent conditions could favour the onset of FA. This involves a great deal of renewed research specialists and experts on the subject to be able to respond appropriately to the diagnostic and therapeutic needs of patients.

\begin{abstract}
Abbreviations
AMA: allergen microarray; APT: atopy patch test; BAT: basophil activation test; DBPCT: double-blind placebo-controlled test; FA: food allergy; FDEIA: food dependent exercise induced anaphylaxis; ISAC: immuno-sorbent allergen chip; LTP: lipid transfer protein; MBD: molecular-based diagnosis,; NSAIDs: non-steroidal anti-inflammatory drugs; OPT: oral provocation test; PR-10: pathogenesis-related-10; P + P: prick + prick; SBT-OPT: single-blind placebo oral provocation test; slgE: specific lgE; SPT: skin prick test; tlgE: total lgE.
\end{abstract}

\section{Authors' contributions}

DM conceived the review and coordinated the other contributors; DM, GM, $V P, E N, M P, M C, C C, M R Y, D S, R P$ contributed to literature review process and to the final version of the manuscript. MDG provided the final revision of the manuscript. All authors read and approved the final manuscript.

\section{Authors' information}

DM is the current Food Allergy Study Group (ATI-Area Tematica di Interesse) Chairperson of the Italian Society of Allergy, Asthma and Clinical Immunology (SIAAIC). VP is the current Food Allergy Study Group (ATI-Area Tematica di Interesse) Secretary of the Italian Society of Allergy, Asthma and Clinical Immunology (SIAAIC). MC is the current Junior Members Chairperson of the Italian Society of Allergy, Asthma and Clinical Immunology (SIAAIC). RP is the current Immunodeficiencies Study Group (ATI-Area Tematica di Interesse) Chairperson of the Italian Society of Allergy, Asthma and Clinical Immunology (SIAAIC). MDG is the current vice-President of the Italian Society of Allergy, Asthma and Clinical Immunology (SIAAIC).

\section{Author details}

${ }^{1}$ SS Allergology and Clinical Immunology, S. Giovanni di Dio Hospital, Florence, Italy. ${ }^{2}$ Respiratory Diseases and Allergy, University of Genoa, IRCCS AOU S. Martino-IST, Genoa, Italy. ${ }^{3}$ Allergology and Immunology Unit, IRCCS Ca' Granda, Osp. Maggiore Policlinico, Milan, Italy. ${ }^{4}$ Servizio di Allergologia,
Policlinico Gemelli, Rome, Italy. ${ }^{5}$ Allergy Unit, Verona University and General Hospital, Verona, Italy. ${ }^{6}$ Department of Allergy and Clinical Immunology, IRCCS San Raffaele Hospital, Milan, Italy. ${ }^{7}$ Department of Medicine and Science of Ageing, G. d'Annunzio University, Chieti, Italy. ${ }^{8}$ Unit of Allergy and Immunotoxicology, CeSi, "G. d'Annunzio" University Foundation, Chieti, Italy.

\section{Acknowledgements}

The authors would like to thank all the members of the Food Allergy Study Group (ATI-Area Tematica di Interesse) of the Italian Society of Allergy, Asthma and Clinical Immunology: Giovanna Alaimo, Valerio Beltrami, Elisa Boni, Maria Bova, Luciana Broccucci, Alessandro Buonomo, Maria Filomena Caiaffa, Stefania Capretti, Mario Caringi, Gloria Castiglioni, Maria Rosaria Cavaluzzo, Michele Cipullo, Pasquale Comberiati, Giovanni Coniglio, Mariaelisabetta Conte, Simona D’Alò, Corrado Danzi, Tiziana De Pasquale, Maria Distaso, Vito Ditta, Maria Pia Dolcher, Paola Fantini, Carmelo Genovese, Paolo Giaccone, Claudio Giagnoni, Davide Grataroli, Ilenia Illuminati, Selene Imbesi, Marco Ispano, Maria Stefania Leto Barone, Fabio Lodi Rizzini, Michela Maggioletti, Diomira Magliacane, Roberto Manetti, Irene Martignago, Ilaria Massaro, Marina Mauro, Valerie Melli, Paolo Meloni, Francesca Mero, Elisa Meucci, Sabrina Mietta, Manlio Milanese, Michela Mineni, Ambra Modiano, Paolo Morandi, Franco Nebiolo, Elisa Olivieri, Angelo Passaleva, Giampiero Patriarca, Giuseppe Pingitore, Alessio Pirino, Celestino Pucci, Cristina Quecchia, Francesca Racca, Erminia Ridolo, Marco Saletti, Gilda Varricchi, Maria Teresa Ventura, Alice Vignoli.

The authors also would like to thank Helen Farrel who provided medical writing services.

\section{Compliance with ethical guidelines}

\section{Competing interests}

The authors declare that they have no competing interests.

Received: 1 September 2015 Accepted: 15 September 2015

Published online: 05 October 2015

\section{References}

1. Lack G. Epidemiologic risks for food allergy. J Allergy Clin Immunol. 2008;121:1331-6.

2. de Blok BM, Vlieg-Boerstra BJ, Oude Elberink JN, Duiverman EJ, DunnGalvin A, Hourihane JO, et al. A framework for measuring the social impact of food allergy across Europe: a EuroPrevall state of the art paper. Allergy. 2007;62:733-7.

3. Boyce JA, Assa'ad A, Burks AW, Jones SM, Sampson HA, Wood RA, et al. Guidelines for the diagnosis and management of food allergy in the United States: summary of the NIAID-sponsored expert panel report. Nutr Res. 2011;31:61-75.

4. DunnGalvin A, Dubois AE, Flokstra-de Blok BM, Hourihane JO. The effects of food allergy on quality of life. Chem Immunol Allergy. 2015;101:235-52.

5. Romano A, Di Fonso M, Giuffreda F, Papa G, Artesani MC, Viola M. Fooddependent exercise-induced anaphylaxis: clinical and laboratory findings in 54 Subjects. Int Arch Allergy Immunol. 2001;125:264-72.

6. Rowlands D, Tofte SJ, Hanifin JM. Does food allergy cause atopic dermatitis? Food challenge testing to dissociate eczematous from immediate reactions. Dermatol Ther. 2006;19:97-103.

7. Burks W. Skin manifestations of food allergy. Pediatrics. 2003;111:1617-24.

8. Chehade M, Sampson HA. The role of lymphocytes in eosinophilic gastrointestinal disorders. Immunol Allergy Clin North Am. 2009;29:149-58.

9. Nowak-Wegrzyn A, Sampson HA, Wood RA, Sicherer SH. Food proteininduced enterocolitis syndrome caused by solid food proteins. Pediatrics. 2003;111:829-35.

10. Furuta GT, Liacouras CA, Collins MH, Gupta SK, Justinich C, Putnam PE, et al. Eosinophilic esophagitis in children and adults: a systematic review and consensus recommendations for diagnosis and treatment. Gastroenterology. 2007;133:1342-63.

11. Kondo Y, Urisu A. Oral allergy syndrome. Allergol Int. 2009;58:485-91.

12. James JM. Respiratory manifestations of food allergy. Pediatrics. 2003;111:1625-30. 
13. Sicherer SH, Leung DY. Advances in allergic skin disease, anaphylaxis, and hypersensitivity reactions to foods, drugs, and insects in 2014. J Allergy Clin Immunol. 2015;135:357-67.

14. Sampson HA, Munoz-Furlong A, Bock SA, Schmitt C, Bass R, Chowdhury $B A$, et al. Symposium on the definition and management of anaphylaxis: summary report. J Allergy Clin Immunol. 2005;115:584-91.

15. Nettis E, Colanardi MC, Ferrannini A, Tursi A. Sodium benzoate-induced repeated episodes of acute urticaria/angio-oedema: randomized controlled trial. Br J Dermatol. 2004;151:898-902.

16. Park HW, Park CH, Park SH, Park JY, Park HS, Yang HJ, et al. Dermatologic adverse reactions to 7 common food additives in patients with allergic diseases: a double-blind, placebo-controlled study. J Allergy Clin Immunol. 2008:121:1059-61.

17. Jones SM. The spectrum of allergic reactions to foods. In: Metcalfe DD, Sampson HA, Simon RA, editors. Food allergy: adverse reactions to foods and food additives, vol. 4. Malden: Wiley-Blackwell; 2008. p. 101-9.

18. Braga M, Quecchia C, Perotta C, Timpini A, Maccarinelli K, Di Tommaso L, Di Gioacchino M. Systemic nickel allergy syndrome: nosologic framework and usefulness of diet regimen for diagnosis. Int J Immunopathol Pharmacol. 2013;26:707-16.

19. Ballmer-Weber BK. Food allergy in adolescence and adulthood. Chem Immunol Allergy. 2015;101:51-8.

20. Canonica GW, Ansotegui IJ, Pawankar R, Schmid-Grendelmeier P, van Hage M, Baena-Cagnani CE, et al. A WAO-ARIA-GA2LEN consensus document on molecular-based allergy diagnostics. World Allergy Organ J. 2013;6:17.

21. Asero R. In patients with LTP syndrome food-specific IgE show a predictable hierarchical order. Eur Ann Allergy Clin Immunol. 2014;46:142-6.

22. Pali-Schöll I, Herzog R, Wallmann J, Szalai K, Brunner R, Lukschal A, et al. Antacids and dietary supplements with an influence on the gastric $\mathrm{pH}$ increase the risk for food sensitization. Clin Exp Allergy. 2010;40:1091-8.

23. Ciprandi G, Cavallucci E, Cuccurullo F, Di Gioacchino M. Helminthic infection as a factor in new-onset coffee allergy in a father and daughter. J Allergy Clin Immunol. 2008;121:773-4.

24. Muñoz-Cano R, Pascal M, Bartra J, Picado C, Valero A, Kim DK, et al. Distinct transcriptome profiles differentiate nonsteroidal anti-inflammatory drug-dependent from nonsteroidal anti-inflammatory drug-independent food-induced anaphylaxis. J Allergy Clin Immunol. 2015;. doi:10.1016/j. jaci.2015.05.042

25. Ben-Shoshan M, Soller L, Harrington DW, Knoll M, La Vieille S, Fragapane J, et al. Eczema in early childhood, sociodemographic factors and lifestyle habits are associated with food allergy: a nested case-control study. Int Arch Allergy Immunol. 2015;166:199-207.

26. Stelmach I, Majak P, Jerzynska J, Podlecka D, Stelmach W, Polańska K, et al. The effect of prenatal exposure to phthalates on food allergy and early eczema in inner-city children. Allergy Asthma Proc. 2015;36:72-8.

27. Wüthrich B. History of food allergy. Chem Immunol Allergy. 2014;100:109-19.

28. Sampson HA, Aceves S, Bock SA, James J, Jones S, Lang D, et al. Food allergy: a practice parameter update-2014. J Allergy Clin Imm. 2014;134:1016-25.

29. Sicherer SH, Sampson HA. Food allergy: epidemiology, pathogenesis, diagnosis, and treatment. J Allergy Clin Immunol. 2014;133:291-307.

30. de Silva D, Geromi M, Panesar SS, Muraro A, Werfel T, Hoffmann-Sommergruber K, et al. Acute and long term management of food allergy: systematic review. Allergy. 2014;69:159-67.

31. Sporik R, Hill DJ, Hosking CS. Specificity of allergen skin testing in predicting positive open food challenges to milk, egg and peanut in children. Clin Exp Allergy. 2000;30:1540-6.

32. Du Toit G, Santos A, Roberts G, Fox AT, Smith P, Lack G. The diagnosis of IgE-mediated food allergy in childhood. Pediatr Allergy Immunol. 2009;20:309-19.

33. Valenta $\mathrm{R}$, Hochwallner $\mathrm{H}$, Linhart $\mathrm{B}$, Pahr S. Food allergies: the basics. Gastroenterology. 2015;148:1120-31.

34. Vlieg-Boerstra BJ, van de Weg WE, van der Heide S, Dubois AE. Where to prick the apple for skin testing? Allergy. 2013;68:1196-8.

35. Henzgen M, Ballmer-Weber BK, Erdmann S, Fuchs T, Kleine-Tebbe J, Lepp U, et al. Skin testing with food allergens. Guideline of the German Society of Allergology and Clinical Immunology (DGAKI), the Physicians' Association of German Allergologists (ADA) and the Society of Pediatric Allergology (GPA) together with the Swiss Society of Allergology. J Dtsch Dermatol Ges. 2008;6:983-8.
36. Cantani A, Micera M. Can skin prick tests provoke severe allergic reactions? Eur Rev Med Pharmacol Sci. 2000;4:145-8.

37. Lieberman JA, Sicherer SH. The diagnosis of food allergy. Am J Rhinol Allergy. 2010;24(6):439-43.

38. Rancé F, Juchet A, Brémont F, Dutau G. Correlations between skin prick tests using commercial extracts and fresh foods, specific lgE, and food challenge. Allergy. 1997;52:1031.

39. Sampson HA. Utility of food-specific lgE concentrations in predicting symptomatic food allergy. J Allergy Clin Immunol. 2001;107:891-6.

40. Katelaris $\mathrm{CH}$. Food allergy and oral allergy or pollen-food syndrome. Curr Opin Allergy Clin Immunol. 2010;10:246-51.

41. Price A, Ramachandran S, Smith GP, Stevenson ML, Pomeranz MK, Cohen DE. Oral allergy syndrome (Pollen-food allergy syndrome). Dermatitis. 2015;26:78-88.

42. Allergome Database. http://www.allergome.org/script/search_step2. php?action=all_allergen. Accessed 30 Aug 2015.

43. Radauer C, Nandy A, Ferreira F, Goodman RE, Larsen JN, Lidholm J, et al. Update of the WHO/IUIS Allergen Nomenclature Database based on analysis of allergen sequences. Allergy. 2014;69:413-9.

44. Gomez F, Aranda A, Campo P, Diaz-Perales A, Blanca-Lopez N, Perkins J, et al. High prevalence of lipid transfer protein sensitization in apple allergic patients with systemic symptoms. PLoS One. 2014;9:e107304.

45. Kollmann D, Geroldinger-Simic M, Kinaciyan T, Huber H, Ebner C, Lidholm J, Bohle B. Recombinant Mal d 1 is a reliable diagnostic tool for birch pollen allergen-associated apple allergy. J Allergy Clin Immunol. 2013;132:1008-10.

46. Ruiter B. Shreffler WG Innate immunostimulatory properties of allergens and their relevance to food allergy. Semin Immunopathol. 2012;34:617.

47. Pastorello EA, Farioli L, Pravettoni V, Scibilia J, Mascheri A, Borgonovo L, et al. Prup p3-sensitized Italian peach-allergic patents are less likely to develop severe symptoms when also presenting IgE Antibodies to Pru P1 and Pru P 4. Int Arch Allergy Immunol. 2011;156:362-72.

48. Mascheri A, Farioli L, Pravettoni V, Piantanida M, Stafylaraki C, Scibilia J, et al. Hypersensitivity to tomato (Lycopersicon esculentum) in peachallergic patients: rPrup 3 and rPrup 1 are predictive of symptom severity. J Investig Allergol Clin Immunol. 2015;25:183-9.

49. Santos A, Van Ree R. Profilins: mimickers of allergy or relevant allergens? Int Arch Allergy Immunol. 2011;155:191-204.

50. Fernandez-Rivas M, Bolhaar S, González-Mancebo E, Asero R, van Leeuwen A, et al. Apple allergy across Europe: how allergen sensitization profiles determine the clinical expression of allergies to plant foods. J Allergy Clin Immunol. 2006;118:481-8.

51. Asero R. Lipid transfer protein cross-reactivity assessed in vivo and in vitro in the office: pros and cons. J Investig Allergol Clin Immunol. 2011;21:129-36.

52. Cudowska B, Kaczmarski M, Restani P. Lipid transfer protein in diagnosis of birch-apple syndrome in children. Immunobiology. 2008;213:89-96.

53. van Ree R, Fernández-Rivas M, Cuevas M, van Wijngaarden M, Aalberse RC. Pollen-related allergy to peach and apple: an important role for profilin. J Allergy Clin Immunol. 1995;95:726-34.

54. Asero R, Mistrello G, Roncarolo D, Amato S, Zanoni D, Barocci F, et al. Detection of clinical markers of sensitization to profilin in patients allergic to plant-derived foods. J Allergy Clin Immunol. 2003;112:427-32.

55. Melioli G, Spenser C, Reggiardo G, Passalacqua G, Compalati E, Rogkakou A, Riccio AM, Di Leo E, Nettis E, Canonica GW. Allergenius, an expert system for the interpretation of allergy microarray results. World Allergy Organ J. 2014;7(1):15

56. Beyer K, Teuber SS. Food allergy diagnostics: scientific and unproven procedures. Curr Opin Allergy Clin Immunol. 2005;5:261-6.

57. Sicherer SH. Food allergy: when and how to perform oral food challenges. Pediatr Allergy Immunol. 1999;10:226-34.

58. Franxman TJ, Howe L, Teich E, Greenhawt MJ. Oral food challenge and food allergy quality of life in caregivers of children with food allergy. J Allergy Clin Immunol Pract. 2015;3:50-6.

59. O'Keefe AW, De Schryver S, Mill J, Mill C, Dery A, Ben-Shoshan M. Diagnosis and management of food allergies: new and emerging options: a systematic review. J Asthma Allergy. 2014;7:141-64.

60. Macchia D, Capretti S, Cecchi L, Colombo G, Di Lorenzo G, Fassio F, et al. Position statement: in vivo and in vitro diagnosis of food allergy in adults. It J Allergy Clin Immunol. 2011;21:57-72. 
61. Mori F, Pucci N, Rossi ME, de Martino M, Azzari C, Novembre E. Oral desensitization to milk: how to choose the starting dose. Pediatr Allergy Immunol. 2010;21:e450-3.

62. Wood RA. Diagnostic elimination diets and oral food provocation. Chem Immunol Allergy. 2015;101:87-95.

63. Bindslev-Jensen C. Standardization of double-blind, placebo-controlled food challenges. Allergy. 2001;56(Suppl 67):75-7.

64. Sabato V, van Hengel AJ, De Knop KJ, Verweij MM, Hagendorens MM, Bridts $\mathrm{CH}$, et al. Human basophils: a unique biological instrument to detect the allergenicity of food. J Investig Allergol Clin Immunol. 2011;21:179-84.

65. Hoffmann HJ, Santos AF, Mayorga C, Nopp A, Eberlein B, Ferrer M, et al. The clinical utility of basophil activation testing in diagnosis and monitoring of allergic disease. Allergy. 2015. doi:10.1111/all.12698.

66. Vassilopoulou E, Karathanos A, Siragakis G, Giavi S, Sinaniotis A, Douladiris $\mathrm{N}$, et al. Risk of allergic reactions to wine, in milk, egg and fish-allergic patients. Clin Transl Allergy. 2011;1:10.

67. Santos AF, Du Toit G, Douiri A, Radulovic S, Stephens A, Turcanu V, et al. Distinct parameters of the basophil activation test reflect the severity and threshold of allergic reactions to peanut. J Allergy Clin Immunol. 2015;135:179-86.

68. Glaumann S, Nilsson C, Johansson SG, Asarnoj A, Wickman M, Borres MP, et al. Evaluation of basophil allergen threshold sensitivity (CD-sens) to peanut and Ara h 8 in children IgE-sensitized to Ara h 8. Clin Mol Allergy. 2015;13:5.

69. Rolland JM, Apostolou E, Deckert K, de Leon MP, Douglass JA, Glaspole IN et al. Potential food allergens in wine: double-blind, placebo-controlled trial and basophil activation analysis. Nutrition. 2006;22:882-8.
70. Kim JH, An S, Kim JE, Choi GS, Ye YM, Park HS. Beef-induced anaphylaxis confirmed by the basophil activation test. Allergy Asthma Immunol Res. 2010;2:206-8.

71. Nucera E, Pecora V, Buonomo A, Rizzi A, Aruanno A, Pascolini L, et al. Utility of basophil activation test for monitoring the acquisition of clinical tolerance after oral desensitization to cow's milk: Pilot study. United European Gastroenterol J. 2015;3:272-6.

72. Senna G, Bonadonna P, Schiappoli M, Leo G, Lombardi C, Passalacqua G. Pattern of use and diagnostic value of complementary/alternative tests for adverse reactions to food. Allergy. 2005;60:1216-7.

73. Heffler E, Minciullo PL, Fassio F, Rossi FW, Patafi M, Mondino M, et al. Pertinence of requested allergy consultations for suspected food allergy/ intolerance. A Junior Members working group of Italian Society of Allergy and Clinical Immunology (SIAIC) multicenter study. J Allergy Clin Immunol. 2011;21:18-24.

74. Semizzi M, Senna G, Crivellaro M, Rapacioli G, Passalacqua G, Canonica WG, et al. A double-blind, placebo-controlled study on the diagnostic accuracy of an electrodermal test in allergic subjects. Clin Exp Allergy. 2002;32:928-32.

75. Garrow JS. Kinesiology and food allergy. Br Med J (Clin Res Ed). 1988;296:1573-4.

76. Jewett $\mathrm{DL}$, Fein $\mathrm{G}$, Greenberg MH. A double-blind study of symptom provocation to determine food sensitivity. N Engl J Med. 1990;323:429-33.

77. Terr Al. The cytotoxic test. West J Med. 1983;139:702-3.

78. Senna G, Passalacqua G, Lombardi C, Antonicelli L. Position paper: controversial and unproven diagnostic procedures for food allergy. Eur Ann Allergy Clin Immunol. 2004;36:139-45.

\section{Submit your next manuscript to BioMed Central and take full advantage of:}

- Convenient online submission

- Thorough peer review

- No space constraints or color figure charges

- Immediate publication on acceptance

- Inclusion in PubMed, CAS, Scopus and Google Scholar

- Research which is freely available for redistribution

Submit your manuscript at

www.biomedcentral.com/submit

C BioMed Central 